\title{
DIAGNOSTIC UTILITY OF SATB2 IN METASTATIC COLORECTAL CARCINOMA
}

\author{
Sammeen Salim, Muhammad Asif, Rabia Ahmed, Hafeez Ud Din, Muhammad Tahir Khadim, Gazala Sadaf \\ Armed Forces Institute of Pathology/National University of Medical Sciences (NUMS) Rawalpindi Pakistan
}

\begin{abstract}
Objective: To evaluate the diagnostic utility of SATB2 in detecting CRC origin for patients presenting with metastatic carcinomas.

Study Design: Cross sectional, comparative study.

Place and Duration of Study: Study was conducted at department of Histopathology, Armed Forces Institute of Pathology, Rawalpindi, from Jan 2019 to Jun 2019.

Methodology: Already diagnosed 68 cases of metastatic carcinoma of various origins with unknown primary were retrieved from tumour registry. Paraffin embedded blocks were taken and a panel of Immunohisto-chemistry was applied which includes CK7, CK20, CDX2 and SATB2. Positivity of SATB2 alone, and combination of SATB2 with CK 7, CK20 and CDX2 was evaluated.

Results: Out of 68 metastatic adenocarcinoma cases 28(41\%) had positive SATB2 expression and $38(56 \%)$ were negative. All the positive SATB2 cases had 100\% (28/28) expression with CDX2, 93\% (26/28) of the cases had expression with CK20 and 21\% (6/28) of the cases showed expression of CK7. The sensitivity of SATB2 alone was $100 \%$ in metastatic colorectal carcinoma as compared to CK20 and CDX2. The sensitivity of SATB2 in combination with CDX2 (100\%) and CK20 (93\%) in comparison to the double combination of CK20 and CDX2 (93\%). The sensitivity of SATB2 and CK20 combination was 93\%, which was equal to CK20 and CDX2 combination.

Conclusion: Our results show that SATB2 is a sensitive marker in the IHC panel for cases with suspected metastasis from colorectal region.
\end{abstract}

Keywords: CDX2, CK7, CK20, mCRC, SATB2.

This is an Open Access article distributed under the terms of the Creative Commons Attribution License (http://creativecommons.org/licenses/by/4.0), which
permits unrestricted use, distribution, and reproduction in any medium, provided the original work is properly cited.

\section{INTRODUCTION}

In the United States Colorectal carcinoma is the fourth most common cancer and the second leading cause of cancer death ${ }^{1}$. The overall prevalence of colorectal cancer in Pakistan is 5\%2. The identification of colonic adenocarcinoma in the colon rarely cause diagnostic difficulties; however, approximately $3-5 \%$ of the cases pre-sents as metastatic carcinoma from unknown primary and at times it becomes difficult to find the primary site $^{3}$. Colorectal carcinoma (CRC) is a globally important health concern due to its high prevalence and mortality rates. Every year, 1 million individuals are diagnosed with CRC and more than 500,000 patients die from it ${ }^{4}$. An estimated 3-5\% of all cancer cases clinically manifest with a metastasis of an unknown primary tumor ${ }^{5}$. Colon

Correspondence: Dr Sammeen Salim, Dept of Histopathology, Armed Forces Institute of Pathology, Rawalpindi Pakistan Received: 25 Jun 2019; revised received: 11 Jun 2019; accepted: 27 Aug 2019 cancer most often spreads to the liver, but it can also spread to other places like the lungs, brain, peritoneum (the lining of the abdominal cavity), or to distant lymph nodes. Diagnostic difficulties arises in detecting metastatic colorectal cracinomas at different sites with unknown primary origin. In metastatic cases that morphologically mimic primary tumors, a suitable immunohistochemical (IHC) panel is selected for the diagnosis based on the histological morphology of the tumor 6,7 . However, the primary focus cannot be identified in a majority of the cases, although immuno-histochemical methods are commonly used $^{8}$. Because different immunohistochemical markers can be positive in variety of normal and neoplastic conditions. Im-munohistochemical markers are antibodies which binds to specific proteins in the tissue and help in the identification of that tissue. There are variety of different immunohistochemical markers which are specific for various tissue types. A Immunohisto- 
chemical marker can be expressed in more than one tissue types. The selection of antibodies that recognize the target proteins is of the utmost importance while selecting the immu-nohistochemical panel. However, very few of these antibodies, which are routinely used for pathology, are expressed in specific cell types. The prostate specific antigen that marks the prostate glandular cells, thyroglobulin that marks the thyroid glandular cells, and the glial fibrillary acidic protein that marks astrocytes are some of these antibodies $^{6}$. On the other hand, a majority of antibodies have limited diagnostic specificity since they are expressed in more than one cell type. The most commonly used immuno-histochemical markers in metastatic tumors that originate from the colon consist of Cytokeratin 20 (CK20) and Caudal type homeobox 2 (CDX2) 6,7,9.

But these immunohistochemical markers do not have high specificity because both can be expressed in tumors other than colon carcinoma. CK20 can also be expressed in urothelial epithelial and epidermal Merkel cells in addition to gastrointestinal system tumors ${ }^{10}$. CDX2 can also be positive in gastric carcinoma and ovarian mucinous tumors. Diffuse expression is detected in $85-100 \%$ of the metastatic cases of colonic origins with CK20 and in 61-100\% of the cases with colon carcinoma with CDX211-14. SATB2 (The special AT-rich sequencebinding protein 2) is the new DNA binding protein and nuclear transcription factor with a length of 733 amino acids. SATB2 is associated with gene trans-cription and chromatin remodeling. SATB2 shows a specific mode of expression and is expressed in nuclei of the cells. Normally SATB2 is expressed in brain, at the site of bone formation, gut, colon, kidney and lymphoid cells. Previous studies demonstrated that SATB2 plays an important role in brain development, craniofacial modelling and osteoblast differentiation ${ }^{15}$. Immunohistochemical studies showed that SATB2 is strongly expressed not only in the normal and neoplastic osteoblastic tissue, but also in the normal colorectal and appendiceal epithelia $^{16}$. In normal epithelial tissues, the SATB2 protein is specifically expressed in the nuclei of the lower gastrointestinal (GI) tract epithelial cells. It is also shown that the SATB2 protein was expressed in non-epithelial cell types (in some lymphoid cells, testicular germ cells and some neurons in the central nervous system). The elective expression of SATB2 in the lower GI tract implies that it can be used as a diagnostic marker for colorectal carcinoma. Therefore, this potential diagnostic biomarker has been analyzed in many colorectal carcinoma and other cancer types ${ }^{17}$. The transcription factor SATB2 was recently identified as a potential marker of high specificity in colorectal adenocarcinoma when used in combination with immunomarker CK20 and CDX218. This study investigates the SATB2, CK7,CK20 and CDX2 expression results in 68 cases with metastatic adenocarcinoma with unknown primary. The aim is to evaluate the diagnostic value of SATB2 alone, and the double and triple combinations of SATB2 with CK7,CK20 and CDX2.

\section{METHODOLOGY}

Present study was approved by the Institutional Review Board. Surgical pathology record of histopathology cases were retrieved for metastatic carcinomas with unknown primary. The cross-sectional study was conducted at the department of Histopathology, Armed Forces Institute of Pathology, Rawalpindi, Pakistan over a period of six months from January 2019 to June 2019. Already diagnosed 68 cases of metastatic carcinoma of various origin with unknown primary were retrieved from tumor registry. These included biopsies from different sites including specimens from lumbar region, omentum, sacrum, gall bladder, urinary bladder, lymph node, ribs, iliac bone, ileum, pancreas, chest wall, Ovary and abdominal wall. Cases with primary adenocarcinomas and malignancies other than adenocarcinoma were excluded. The hematoxylin and eosin slides were retrieved to confirm the diagnosis. The formalin fixed, paraffin embedded blocks were taken and a panel of immunohistochemistry was applied which includes Ck7, Ck20, CDX2 and SATB $2.4 \mu \mathrm{m}$ thick sections were generated from whole tissue blocks for immunohistochemical staining on a Ventana Benchmark-XT 
automated stainer using the Ventanaultra View DAB detection kit. Positive controls (nor-mal colonic mucosa as positive control for SATB2, CDX2, CK20; normal gastric mucosa as positive control for CK7) were included for each run of immunostains. Only nuclear staining was considered positive for SATB2 and CDX2. Cyto-plasmic and/or membranous staining was con-sidered positive for CK7 and CK20. The interpretation of immunohistochemical markers was done by two pathologists. The data was entered and statistical analysis was done by spss version 21 . The relationships between variables were determined using the Fisher exact test. A $p$-value of $<0.05$ was considered statistically significant. Sensitivity was calculated as true-positive/(true-positive +falsenegative) and specificity is cal-culated as truenegative/ (true-negative+false-positive).

\section{RESULTS}

In the present study a total of 68 cases of metastatic adenocarcinoma with unknown primary were investigated. Age and gender of patients were recorded. Forty four $(64.7 \%)$ were male and

Table-I: Number of cases with different metastatic sites.

\begin{tabular}{l|c}
\hline Metastatic Sites & n (\%) \\
\hline Liver & $30(44.1)$ \\
\hline Lymph Node & $2(2.9)$ \\
\hline Ribs & $2(2.9)$ \\
\hline Iliac bone & $2(2.9)$ \\
\hline Lumbar Region & $4(5.9)$ \\
\hline Omentum & $4(5.9)$ \\
\hline Sacrum & $4(5.9)$ \\
\hline Gall bladder & $4(5.9)$ \\
\hline Urinal Bladder & $4(5.9)$ \\
\hline Ovary & $4(5.9)$ \\
\hline Ileum & $2(2.9)$ \\
\hline Pancreas & $2(2.9)$ \\
\hline Chest Wall & $2(2.9)$ \\
\hline Abdominal Wall & $2(2.9)$ \\
\hline Total & $68(100.0)$ \\
\hline
\end{tabular}

$24(35.3 \%)$ were females having mean age of 58.46 Years and S.D. of 12.97. Out of these 68 patients metastatic adenocarcinoma was the primary diagnosis in various sites which includes $30(44.1 \%)$ liver, 4 (5.9\%) lumbar region, 4 (5.9\%) omentum, 4 (5.9\%) sacrum, 4 (5.9\%) gall bladder, $4(5.9 \%)$ urinary bladder, 2 (2.9\%) lymph Node, $2(2.9 \%)$ ribs, 2 (2.9\%) iliac bone, 4 (5.9\%) ovary, $2(2.9 \%)$ ileum, $2(2.9 \%)$ pancreas, $2(2.9 \%)$ chest wall and 2 (2.9\%) abdominal wall (table-I). Out of total 68 cases with metastatic carcinoma $28(41 \%)$ had positive SATB2 expressions and 40 (58.8\%) had negative SATB2 expression (table-II \& III).

Table-II: Expression of different immunohistochemical markers.

\begin{tabular}{|c|c|c|}
\hline & $\begin{array}{l}\text { Metastatic } \\
\text { Colorectal } \\
\text { Carcinoma } \\
\quad(n=28)\end{array}$ & $\begin{array}{c}\text { Metastatic } \\
\text { Adenocarcinoma } \\
\text { (other Sites) } \\
(n=40)\end{array}$ \\
\hline \multicolumn{3}{|l|}{ SATB2 } \\
\hline Positive & 28 & 0 \\
\hline Negative & 0 & 40 \\
\hline \multicolumn{3}{|l|}{ CDX2 } \\
\hline Positive & 28 & 8 \\
\hline Negative & 0 & 32 \\
\hline \multicolumn{3}{|l|}{ CK20 } \\
\hline Positive & 26 & 10 \\
\hline Negative & 2 & 30 \\
\hline \multicolumn{3}{|l|}{ CK7 } \\
\hline Positive & 6 & 32 \\
\hline Negative & 22 & 8 \\
\hline
\end{tabular}

Thus metastatic adenocarcinoma with primary likely from colorectal region was found in $28(41.2 \%)$ while metastatic adenocarcinoma with primary from sites other than colorectal region was found in $40(58.8 \%)$ patients. It was found that all the positive SATB2 cases had 100\% (28/ 28) expression with CDX2 with significant $p$ value i.e (0.00), 93\% (26/28) of the cases had expression with CK20 with significant $p$-value i.e. $(0.00)$ and $21 \%(6 / 28)$ of the cases had expression with CK7 (table-IV). The sensitivity of SATB2 alone was $100 \%$ in metastatic colorectal carcinoma as compared to CK20 and CDX2. The sensitivity of SATB2 in combination with CDX2 $(100 \%)$ and CK20 (93\%) in comparison to the double combination of CK20 and CDX2 (93\%). The sensitivity of SATB2 and CK20 combination was $93 \%$, which was equal to CK20 and CDX2 combination. 
Table-III: Description of sample.

\begin{tabular}{l|c}
\hline \multicolumn{1}{l}{ Metastatic Colorectal Carcinoma } & $\mathbf{n = 2 8}$ \\
\hline Gender & 20 \\
\hline Male & 8 \\
\hline \multicolumn{1}{l}{ Female } & \\
\hline Age (Years) & 1 \\
\hline $18-29$ & 7 \\
\hline $30-44$ & 7 \\
\hline $45-59$ & 13 \\
\hline $60-$ Above & \\
\hline Specimen Site & 8 \\
\hline Liver & 2 \\
\hline Lymph Node & 2 \\
\hline Ribs & 2 \\
\hline Growth Lumbar Region & 2 \\
\hline Sacral Mass & 2 \\
\hline Gal bladder & 4 \\
\hline Bladder Growth & 2 \\
\hline Adnaxel Mass & 2 \\
\hline Ileum & 2 \\
\hline Pancreas &
\end{tabular}

Table-IV: Double and triple combinations of SATB2 with CK7, CK20 and CDX2.

\begin{tabular}{l|c|c}
\hline & $\begin{array}{c}\text { Metastatic } \\
\text { Colorectal } \\
\text { Carcinoma } \\
(\mathbf{n = 2 8 )}\end{array}$ & $\begin{array}{c}\text { Metastatic } \\
\text { Adenocarcinoma } \\
\text { (Other sites) } \\
(\mathbf{n = 4 0 )}\end{array}$ \\
\hline SATB2+/CK20+ & 26 & 2 \\
\hline SATB2+/CDX2+ & 28 & 2 \\
\hline SATB2+/CK7+ & 6 & 2 \\
\hline $\begin{array}{l}\text { SATB2+/CK20+ } \\
\text { /CDX2+ }\end{array}$ & 26 & 2 \\
\hline $\begin{array}{l}\text { SATB2- } \\
\text { /CK20+/CK7+ }\end{array}$ & 0 & 10 \\
\hline CK20+/CDX2+ & 26 & 8 \\
\hline
\end{tabular}

\section{DISCUSSION}

The purpose of this study was to evaluate SATB2 expression in metastatic colorectal cancer in biopsies suggesting metastasis from unknown primary. The mass in the colon does not cause diagnostic difficulties, howvever problem arises when it presents at metastatic sites with unknown primary. So we have checked the diagnostic utility of SATB2 in detecting colorectal origin and compared it with panel of immunohistochemical markers which are routinely used when we suspect metastatic carcinoma from colorectal origin. In our setup we routinely used a panel of immunohistochemical markers when suspecting metastasis from colorectal origin which includes CK7, CK20 and CDX2. Our results are comparable with other studies in which similar sensitivity with SATB2 expression was observed in colorectal cancer ${ }^{18}$. In our study, SATB2 shows greater sensitivity than CK20 for the diagnosis of metastatic colorectal cancer. The specificity was higher than CK20 expression, suggesting that the combination of SATB2 and CK20 can improve the diagnostic accuracy of colorectal cancer origin. It was also noted that SATB2 showed minimal expression in metastatic adenocarcinoma other that colorectal origin. These findings suggests that if a metastatic adenocarcinoma with unknown primary shows SATB2 expression, it is highly suggestive of colorectal origin. A study carried by Zhang et al. (2018) showed that SATB2 is a promising biomarker for identifying a colorectal origin for liver metastatic adenocarcinoma. His results show $92.2 \%$ sensitivity and $97.8 \%$ sensitivity $^{9}$ for SATB2, 95.1\% sensitivity and 91\% specificity for CK20, and 100\% sensitivity and 85.4\% specificity for CDX2. He further demonstrated that all three immunohistochemical marker panel further improved the detection of metastatic colorectal cancers in liver biopsy tissues.

Another study carried out by Hewedi et al, showed that SATB2 expression has significantly higher sensitivity for the diagnosis of mucinous adenocarcinoma of colorectal origin being $100 \%$ compared with $60 \%$ for CDX2 $(p=0.046)^{19}$. The specificity of CDX2 for the diagnosis of mucinous adenocarcinoma of colorectal origin was significantly higher being 100\% compared with 56.2\% for SATB2 $(p=0.001)$. The positive predictive value of SATB2 and CDX2 for distinguishing mucinous adenocarcinoma of colorectal origin from mucinous carcinomas of other origins was 41.6 and $100 \%$, respectively, whereas the NPV was $100 \%$ for SATB2 and $88.89 \%$ for CDX2.

Similarly in a study by Yang et al, an investigation was carried out on immuno-histochemical expression of SATB2 in a large series of 70 MKTs (metastatic krunkenburg tumours) of various origins in the ovary showed that SATB2 is a sensitive marker for MKT originated from 
appendiceal Adex GCCs having 100\% specificity and sensitivity ${ }^{20}$. Our result shows that the use of SATB2 can be helpful in detecting colorectal origin from unknown primary. Combination of SATB2 with CK20 and CDX2 can further improved the detection of metastatic Colorectal cancers in biopsy with suspecting metastasis from unknown primary. The similarity of our results with prior studies indicates that SATB2 is a diagnostic marker for colorectal cancer and can be used in a immunohistochemical panel use for detection of colorectal origin in metastatic cases.

\section{CONCLUSION}

Our results show that SATB2 is a sensitive marker in the immunohistochemical panel for cases with suspected metastasis from colorectal region. This study shows the diagnostic utility of SATB2 in metastatic colorectal carcinoma and demonstrated that the combination of immunohistochemical markers such as SATB2, CK7, CK20 and CDX2 can further improves the diagnostic accuracy.

\section{CONFLICT OF INTEREST}

This study has no conflict of interest to declare by any author.

\section{REFERENCES}

1. Siegel RL, Miller KD, Jemal A. Cancer statistics, 2016. CA: Cancer J Clinicians 2016; 66(1): 7-30.

2. Idrees R, Fatima S, Abdul-Ghafar J, Raheem A, Ahmad Z. Cancer prevalence in Pakistan: meta-analysis of various published studies to determine variation in cancer figures resulting from marked population heterogeneity in different parts of the country. World J Surg Oncol 2018; 16(1): 129-31.

3. Conner JR, Hornick JL. Metastatic carcinoma of unknown primary: diagnostic approach using immunohistochemistry. Adv Anat Pathol 2015; 22(3): 149-67.

4. Cunningham D, Atkin W, Lenz Hj, Lynch HT, Minsky B, Nordlinger B, et al. Colorectal cancer. Lancet 2010; 375(9719): 1030-47.

5. Pavlidis N, Fizazi K. Carcinoma of unknown primary (CUP). Crit Rev 2009; 69(3): 271-78.
6. Dragomir A, de Wit M, Johansson C, Uhlen M, Pontén F. The role of SATB2 as a diagnostic marker for tumors of colorectal origin: results of a pathology-based clinical prospective study. Am J Clini Pathol 2014; 141(5): 630-38.

7. Montiel DP, Angulo KA, Cantú-de León D, Quevedo LB, Vilchis JC, Montalvo LH. The value of SATB2 in the differential diagnosis of intestinal-type mucinous tumors of the ovary: primary vs metastatic. Ann Diag Pathol 2015; 19(4): 249-52.

8. Pentheroudakis G, Briasoulis E, Pavlidis N. Cancer of unknown primary site: missing primary or missing biology? Oncologist 2007; 12(4): 418-25.

9. Zhang YJ, Chen JW, He XS, Zhang HZ, Ling YH, Wen JH, et al. SATB2 is a promising biomarker for identifying a colorectal origin for liver metastatic adenocarcinomas. E Bio Medicine 2018; 2018(28): 62-69.

10. Moll RSD, Franke WW. Identification of protein IT of the intestinal cytoskeleton as a novel type I cytokeratin with unusual properties and expression patterns. J Cell Biol 1990; 111(1): 567-80.

11. Pinto PB DS, Andrade LA. Metastatic mucinous carcinoma in the ovary: a practical approach to diagnosis to gross aspects and to immunohistochemical evaluation. Int J Gynecol Pathol 2012; 31(1): 313-18.

12. MR. Epithelial neoplasms of the large intestine. In: Odze R, Goldblum JR, editors. Surgical pathology of the GI tract, liver, biliary tract and pancreas. Philadelphia: Saunders. 2009: 597-637.

13. Vang R GA, WuL S. Immunohistochemical expression of CDX2 in primary ovarian mucinous tumors and metastatic mucinous carcinoma involving the ovary: comparison with CK20, and correlation with coordinate expression of CK7. Mod Pathol 2006; 19(1): 1421-28.

14. Werling RW YH, Bachi CE, Gown AM. CDX2, a highly sensitive and specific marker of adenocarcinoma of intestinal origin: an immunohistochemical survey of 476 primary and metastatic carcinoma. Am J Surg Pathol 2003; 27(1): 303-10.

15. Wu L, Chen J, Qin Y, Mo X, Huang M, Ru H, et al. SATB2 suppresses gastric cancer cell proliferation and migration. Tumor Biol 2016; 37(4): 4597-602.

16. Brocato JCM. SAT1 and SAT2 in colorectal cancer. Carcinogenesis 2015; 36(2): 186-91.

17. Magnusson KWM, Brennan DJ, Johnson LB. SATB2 in combination with cytokeratin 20 identifies over $95 \%$ of all colorectal carcinomas. Am J Surg Pathol 2011; 35(1): 937-48.

18. Magnusson K, de Wit M, Brennan DJ, Johnson LB, McGee SF, Lundberg E, et al. SATB2 in combination with cytokeratin 20 identifies over $95 \%$ of all colorectal carcinomas. Am J Surg Pathol 2011; 35(7): 937-48.

19. Hewedi IH, Shakweer MM, Radwan NA. Diagnostic value of the combined use of SATB2 and CDX2 in mucinous carcinoma of colorectal origin. Egyptian J Pathol 2017; 37(1): 112-19.

20. Yang C, Sun L, Zhang L, Zhou L, Zhao M, Peng Y, et al. Diagnostic Utility of SATB2 in Metastatic Krukenberg Tumors of the Ovary. Am J Surg Pathol 2018; 42(2): 160-71. 